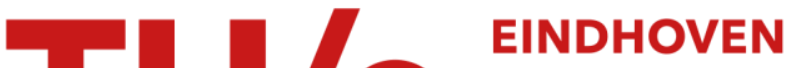

\section{Spin-lock MR enhances the detection sensitivity of superparamagnetic iron oxide particles}

\section{Citation for published version (APA):}

Moonen, R. P. M., van der Tol, P., Hectors, S. J. C. G., Starmans, L. W. E., Nicolaij, K., \& Strijkers, G. J. (2015). Spin-lock MR enhances the detection sensitivity of superparamagnetic iron oxide particles. Magnetic Resonance in Medicine, 74(6), 1740-1749. https://doi.org/10.1002/mrm.25544

DOI:

10.1002/mrm.25544

Document status and date:

Published: 01/12/2015

\section{Document Version:}

Publisher's PDF, also known as Version of Record (includes final page, issue and volume numbers)

\section{Please check the document version of this publication:}

- A submitted manuscript is the version of the article upon submission and before peer-review. There can be important differences between the submitted version and the official published version of record. People interested in the research are advised to contact the author for the final version of the publication, or visit the $\mathrm{DOI}$ to the publisher's website.

- The final author version and the galley proof are versions of the publication after peer review.

- The final published version features the final layout of the paper including the volume, issue and page numbers.

Link to publication

\section{General rights}

Copyright and moral rights for the publications made accessible in the public portal are retained by the authors and/or other copyright owners and it is a condition of accessing publications that users recognise and abide by the legal requirements associated with these rights.

- Users may download and print one copy of any publication from the public portal for the purpose of private study or research.

- You may not further distribute the material or use it for any profit-making activity or commercial gain

- You may freely distribute the URL identifying the publication in the public portal.

If the publication is distributed under the terms of Article 25fa of the Dutch Copyright Act, indicated by the "Taverne" license above, please follow below link for the End User Agreement:

www.tue.nl/taverne

Take down policy

If you believe that this document breaches copyright please contact us at:

openaccess@tue.nl

providing details and we will investigate your claim. 


\title{
Spin-Lock MR Enhances the Detection Sensitivity of Superparamagnetic Iron Oxide Particles
}

\author{
Rik P. M. Moonen, ${ }^{1}$ Pieternel van der Tol, ${ }^{1,2}$ Stefanie J. C. G. Hectors, ${ }^{1}$ \\ Lucas W. E. Starmans, ${ }^{1}$ Klaas Nicolay, ${ }^{1}$ and Gustav J. Strijkers ${ }^{1,3 *}$
}

Purpose: To evaluate spin-lock MR for detecting superparamagnetic iron oxides and compare the detection sensitivity of quantitative $T_{1 p}$ with $T_{2}$ imaging.

Methods: In vitro experiments were performed to investigate the influence of iron oxide particle size and composition on $T_{1 \rho}$. These comprise $T_{1 \rho}$ and $T_{2}$ measurements $\left(B_{0}=1.41 T\right)$ of agar $(2 \%)$ with concentration ranges of three different iron oxide nanoparticles (IONs) (Sinerem, Resovist, and IONMicelle) and microparticles of iron oxide (MPIO). $T_{1 \rho}$ dispersion was measured for a range of spin-lock amplitudes $\left(\gamma B_{1}=6.5-\right.$ $91 \mathrm{kHz})$. Under relevant in vivo conditions $\left(B_{0}=9.4 \mathrm{~T} ; \gamma B_{1}=\right.$ $100-1500 \mathrm{~Hz}), T_{1 \rho}$ and $T_{2}$ mapping of the liver was performed in seven mice pre- and $24 \mathrm{~h}$ postinjection of Sinerem.

Results: Addition of iron oxide nanoparticles decreased $T_{1 \rho}$ as well as the native $T_{1 p}$ dispersion of agar, leading to increased contrast at high spin-lock amplitudes. Changes of $T_{1 p}$ were highly linear with iron concentration and much larger than $T_{2}$ changes. MPIO did not show this effect. In vivo, a decrease of $T_{1 \rho}$ was observed with no clear influence on $T_{1 \rho}$ dispersion. Conclusion: By suppression of $T_{1 \rho}$ dispersion, iron oxide nanoparticles cause enhanced $T_{1 \rho}$ contrast compared to $T_{2}$. The underlying mechanism appears to be loss of lock. Spinlock MR is therefore a promising technique for sensitive detection of iron oxide contrast agents. Magn Reson Med 74:1740-1749, 2015. ( 2014 Wiley Periodicals, Inc.

Key words: Spin-lock MR; iron oxide nanoparticles; rotating frame relaxation; $T_{1 \rho}$ contrast agent; $T_{1 \rho}$ dispersion; superparamagnetic iron oxide particles

\section{INTRODUCTION}

Superparamagnetic iron oxide particles find application as contrast agents for molecular and cellular MRI and are used to facilitate in vivo cell tracking and, among others, inflammation imaging (1-4). The presence of superpara-

${ }^{1}$ Biomedical NMR, Department of Biomedical Engineering, Eindhoven University of Technology, Eindhoven, The Netherlands.

${ }^{2}$ Department of Radiology, Leiden University Medical Center, Leiden, The Netherlands.

${ }^{3}$ Biomedical Engineering and Physics, Academic Medical Center, Amsterdam, The Netherlands.

Grant sponsor: The Center for Translational Molecular Medicine and the Dutch Heart Foundation (PARISk); Grant number: 01-202.

${ }^{*}$ Correspondence to: Dr. Gustav J. Strijkers, Academic Medical Center, Department of Biomedical Engineering and Physics, P.O. Box 22700, 1100 DE Amsterdam, The Netherlands. E-mail: g.j.strijkers@amc.uva.nl

Received 28 August 2014; revised 14 October 2014; accepted 3 November 2014

DOI 10.1002/mrm.25544

Published online 2 December 2014 in Wiley Online Library (wileyonlinelibrary. com).

(c) 2014 Wiley Periodicals, Inc. magnetic iron oxide particles in tissue can be visualized by lowered signal intensity or signal voids in $\mathrm{T}_{2^{-}}$or $\mathrm{T}_{2}{ }^{*}$ weighted imaging. This negative contrast poses challenges to the quantitative assessment of iron concentration; moreover, interpretation is conspicuous because signal voids are often difficult to distinguish from image artifacts. Therefore, considerable effort is put in the development of MRI methods that provide a quantitative readout of the presence of iron oxides. Furthermore, quantitative MR imaging enables standardized comparisons of results between different sites and systems. A common approach to quantitative imaging of iron oxides is by mapping the transversal relaxation time $\mathrm{T}_{2}\left(^{*}\right)(5-10)$.

In this study, we investigated quantitative $\mathrm{T}_{1 \rho}$ measurements for imaging of iron oxide-based contrast agents. We hypothesized that $\mathrm{T}_{1 \rho}$ provides a more sensitive readout of the presence of iron oxides than $\mathrm{T}_{2} . \mathrm{T}_{1 \rho}$ is the longitudinal relaxation in the rotating frame of reference during the application of a radiofrequency (RF) pulse parallel to the magnetization vector. $\mathrm{T}_{1 \mathrm{p}}$-weighted imaging is commonly accomplished using spin-lock MR, for which magnetization is first excited by a $90^{\circ}$ pulse and subsequently subjected to a long, continuous-wave $B_{1}$ spin-lock pulse. Because the $B_{1}$ field is low compared to the $B_{0}$ field, the tissue $T_{1 \rho}$ relaxation is mediated by processes with long correlation times such as proton diffusion, proton exchange, and macromolecular tumbling. These processes can be probed by varying the $B_{1}$ spin-lock field strength; they show up as tissue $\mathrm{T}_{1 \rho}$ dispersion as function of $\mathrm{B}_{1}$ (11-16). The $\mathrm{T}_{1 \rho}$ relaxation is also influenced by the proton off-resonance frequency $(\Delta \omega)$, which introduces a $\mathrm{T}_{1}$ component that depends on the ratio of $\mathrm{B}_{1}$ and $\Delta \omega(17-20)$.

From $\mathrm{T}_{2}$ and $\mathrm{T}_{2}{ }^{*}$ relaxation models, it is known that iron oxides enhance the proton relaxation rate via their local magnetic susceptibility in a particle size-, coating-, and diffusion-dependent manner (21-23). These effects are categorized into several relaxation regimes. Similar mechanisms will influence $\mathrm{T}_{1 \mathrm{\rho}}$ relaxation in the presence of iron oxides. We expected that protons in the static regime will experience off-resonance spin lock in the vicinity of a particle. Protons that diffuse through the magnetic field gradients on the timescale of the spinlock pulse duration, however, were predicted to lose their spin lock because of the fluctuating magnetic field that they experience. We therefore hypothesize that, in the latter diffusion regime, the presence of iron oxides could lead to changes in the tissue $\mathrm{T}_{1 \rho}$ dispersion that are larger than $\mathrm{T}_{2}$ changes, potentially providing a more sensitive readout for the detection of iron oxides. 
Table 1

Relevant Properties of the Iron Oxide Contrast Agents

\begin{tabular}{|c|c|c|c|c|}
\hline & Sinerem & Resovist & ION-Micelle & MPIO \\
\hline Coating & Dextran (42) & Carboxydextran (42) & PEG-lipid & $\begin{array}{c}\text { polystyrene/ } \\
\text { divinylbenzene }\end{array}$ \\
\hline Iron oxide core diameter $(\mathrm{nm})$ & $4-6(43)$ & $4-6(29,44)$ & $25(25)$ & $4-13^{a}$ \\
\hline Hydrodynamic diameter (nm) & $32(25)$ & $62(25)$ & $61(25)$ & $860^{\mathrm{b}}$ \\
\hline Magnetization $\left(\mathrm{Am}^{2} \mathrm{~kg}^{-1} \mathrm{Fe}\right)$ & 71 & 89 & 93 & 105 \\
\hline
\end{tabular}

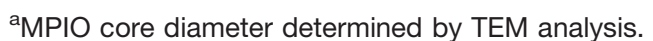

${ }^{\mathrm{b}} \mathrm{MPIO}$ particle diameter provided by manufacturer.

To evaluate this hypothesis, $\mathrm{T}_{1 \rho}$ dispersion curves of agarose gels with various concentrations of superparamagnetic iron oxide micro- and nanoparticles were measured and compared to $\mathrm{T}_{2}$ data. Particles with different iron oxide core size, overall particle size, coating thickness, and composition were selected such that different relaxation regimes could be probed (24). Additionally, mice were injected with an iron oxide contrast agent, and quantitative $T_{1 \rho}$ and $T_{2}$ values of the contrast agentcontaining liver were evaluated.

\section{METHODS}

\section{Contrast Agents, Characterization, and Properties}

Four superparamagnetic iron-oxide contrast agents were chosen for their variation in size and composition: Sinerem (ferumoxtran; Guerbet, Villepoint, France), Resovist (ferucarbotran; Bayer Schering Pharma, Berlin, Germany), iron oxide nanoparticle (ION)-Micelles (homemade (25)), and microparticles of iron oxide (MPIO) (Bangs Laboratories Inc, Fishers, Indiana). Table 1 summarizes relevant characteristics of the particles. Sinerem and Resovist have a similar iron oxide core size (4$6 \mathrm{~nm}$ ) and coating composition (dextran) but a different hydrodynamic diameter; they were chosen to investigate the effect of the coating thickness on $\mathrm{T}_{1 \rho}$ relaxation. IONMicelles have a much larger core size (25 nm), although their hydrodynamic diameter (61 nm) is comparable to that of Resovist ( $62 \mathrm{~nm}$ ), yielding data on the effect of iron oxide core size. The hydrodynamic diameter of MPIO (860 nm) is much larger than the other three particles and it was selected to evaluate $\mathrm{T}_{1 \rho}$ dispersion in the static dephasing regime. In this article, Sinerem, Resovist, and ION-Micelles will be referred to as nanoparticles; MPIO will be referred to as microparticles.

Transmission electron microscopy (TEM) images were acquired with a Tecnai F30ST (FEI, Hillsboro, OR) TEM with field emitter gun operated at $300 \mathrm{kV}$. Cryo-TEM was performed using a cryo-holder at $-170{ }^{\circ} \mathrm{C}$. Saturation magnetization values were determined using a vibrating sample magnetometer (ADE Technologies, Newton, MA)) at room temperature; sample iron concentrations were obtained with inductively coupled plasma atomic emission spectrometry (ICP-AES).

\section{Sample Preparation}

The dilution series for the in vitro nuclear magnetic resonance (NMR) measurements were made in $2 \%$ agar gel because of its tissue-like $\mathrm{T}_{1}$ and $\mathrm{T}_{2}$ properties. Agarose (Sigma-Aldrich, St. Louis, MO) was dissolved in demine- ralized water by heating it to $80{ }^{\circ} \mathrm{C}$, thoroughly mixed with the iron oxide contrast agent solution, and then poured into $5 \mathrm{~mm}-\mathrm{NMR}$ tubes (Bruker BioSpin, Ettlingen, Germany). During the pouring, the tubes were placed in a water bath at $80{ }^{\circ} \mathrm{C}$ to prevent the agar gel from solidifying against the walls of the tubes. Subsequently, the tubes were placed at room temperature to allow the gel to solidify on the bottom of the tube. The iron concentrations $(0,50,125,250$, and $500 \mu \mathrm{M})$ were chosen to be identical for the different contrast agents because it is common practice to assess relaxation enhancement as a function of iron concentration. This procedure consequently results in varying particle concentrations. All samples were made as one single batch, that is, from the same agarose solution.

\section{In Vitro NMR}

All experiments were performed with a tabletop NMR spectrometer operating at $60 \mathrm{MHz} / 1.41 \mathrm{~T}$ (Minispec60, Bruker BioSpin) with a solenoid RF coil, and the sample temperature was maintained at $20{ }^{\circ} \mathrm{C}$. The $\mathrm{T}_{1 \rho}$ sequence consisted of a $90^{\circ}$ excitation pulse directly followed by a continuous wave spin-lock pulse and free induction decay acquisition. In order to assess the $\mathrm{T}_{1 \rho}$ dispersion, the spin-lock amplitude was varied between 6.5 and $91 \mathrm{kHz}$. The spin-lock amplitude could not be set directly and was adjusted by changing the RF attenuation. Using a sample of $2 \%$ agar gel without contrast agent, the $90^{\circ}$ pulse duration was calibrated for each setting of the RF attenuation, and then the spin-lock amplitude $\gamma \mathrm{B}_{1}$ at the set RF attenuation was calculated.

$\mathrm{T}_{1 \rho}$ was determined from exponential fitting of peak signal intensities from 10 different spin-lock durations. Spin-lock durations were logarithmically distributed between a minimum of $1 \mathrm{~ms}$ and a maximum of 10 to 50 $\mathrm{ms}$, with the maximum depending on $\mathrm{B}_{1}$ and hardware restrictions. For comparison with $\mathrm{T}_{1 \rho}, \mathrm{T}_{2}$ was acquired using the Carr-Purcell-Meiboom-Gill (CPMG) sequence with 1024 echoes, $4.6 \mu \mathrm{s} 180^{\circ}$ pulse duration, and a $90^{\circ}-180^{\circ}$ pulse separation of 40 to $500 \mu \mathrm{s}$, resulting in a longest echo time (TE) ranging between 81.92 and $1024 \mathrm{~ms}$. For all in vitro NMR experiments, the repetition time (TR) was set to five times the sample $\mathrm{T}_{1}$, as determined by an inversion recovery experiment.

\section{NMR Data Analysis}

The dispersion data were fitted with an exchange model in order to extrapolate the $\mathrm{T}_{1 \rho}$ curves to a spin-lock amplitude of $0 \mathrm{kHz}$ for direct comparison with $\mathrm{T}_{2}$ acquired with the CPMG sequence. Relaxation behavior 
in agar gels can be approximated by a two-pool exchange model with a free water and a bound water pool. The equation describing a two-pool exchange model assuming negligible dipolar relaxation effects and on or near resonance conditions is (11)

$$
R_{1 \rho}=R_{1} \cos ^{2} \theta+\left(R_{2}^{0}+A \frac{r_{b}}{r_{b}^{2}+\omega_{1}^{2}}\right) \sin ^{2} \theta
$$

with $R_{1 \rho}$ the longitudinal relaxation rate in the rotating frame $\left(\mathrm{T}_{1 \rho}{ }^{-1}\right), \mathrm{R}_{2}^{0}$ the transverse relaxation rate without exchange, $\theta$ the tilt angle of the effective spin-lock field, A $=\mathrm{p}_{\mathrm{f}} \mathrm{p}_{\mathrm{b}} \Delta \omega^{2}$ the product of the fractions of free and bound water multiplied by the square of their frequency difference, $\mathrm{r}_{\mathrm{b}}$ the exchange rate, and $\omega_{1}$ the spin-lock amplitude in $\mathrm{rad} / \mathrm{s}$. Assuming on-resonance spin lock $(\theta=1 / 2 \pi)$ and substituting $\mathrm{r}_{\mathrm{b}}$ with $\frac{1}{\tau_{e x}}$, Equation (1) is rewritten as

$$
\mathrm{R}_{1 \rho}=\mathrm{R}_{2}^{0}+\mathrm{A} \frac{\tau_{\mathrm{ex}}}{1+\omega_{1}^{2} \tau_{\mathrm{ex}}^{2}}
$$

with $\tau_{\text {ex }}$ the exchange time. The dispersion data was fitted to this equation, and $R_{1 \rho}\left(\omega_{1}=0\right)$ was compared to $R_{2}$ measured by the CPMG sequence.

To assess the effect on contrast, the normalized change in $\mathrm{R}_{1 \rho}$,

$$
N \Delta R_{1 \rho}=\frac{R_{1 \rho C A}-R_{1 \rho 0}}{R_{1 \rho 0}}
$$

was determined for each spin-lock amplitude. Here, $\mathrm{R}_{1 \rho \mathrm{CA}}$ is $\mathrm{R}_{1 \rho}$ of the sample with contrast agent, and $\mathrm{R}_{1 \rho 0}$ is $R_{1 \rho}$ of the plain agar sample. Similarly, we define the normalized change in $R_{2}$,

$$
N \Delta R_{2}=\frac{R_{2 C A}-R_{2,0}}{R_{2,0}}
$$

for a quantitative comparison of the change in $R_{1 \rho}$ and $R_{2}$ values in the presence of iron oxide contrast materials.

\section{In Vivo MRI}

Animal experiments were approved by the animal experiment committee of Maastricht University (The Netherlands). Seven female C57bl/6 mice (Charles River, Maastricht, The Netherlands) with 20.5 to 22.3 g bodyweight (BW) were anesthetized with $1 \%$ to $2 \%$ isoflurane. A catheter containing $0.9 \% \mathrm{NaCl}$ with heparin and a $150 \mu \mathrm{l}$ bolus of $50 \mu \mathrm{mol}$ $\mathrm{Fe} / \mathrm{kg}$ BW Sinerem was inserted into the tail vein. Sinerem was chosen for the in vivo experiments because it was one of the three nanoparticles that yielded enhanced contrast in the in vitro experiments. The mice were placed in a supine position on an animal bed with an anesthesia mask and monitored with electrocardiogram (ECG) electrodes attached to the front paws, a balloon respiration sensor, and a rectal temperature probe. The body temperature was maintained at approximately $37^{\circ} \mathrm{C}$ with a warm water pad.

In vivo MRI experiments were performed with a $9.4 \mathrm{~T}$ small animal scanner equipped with a 35-mm-diameter quadrature birdcage RF coil (Bruker BioSpin). An axial slice (field of view $=22 \times 22 \mathrm{~mm}^{2}$, matrix $=220 \times 220$, thickness $=1 \mathrm{~mm}$ ) was positioned just under the dia- phragm to encompass a large section of the liver. The liver was chosen because Sinerem is excreted via the hepatobiliary pathway and will thus accumulate in the liver and spleen. All acquisitions were respiratory-gated and ECG-triggered, with the acquisition window positioned in the end-diastolic phase of the cardiac cycle in order to minimize motion artifacts caused by cardiac and respiratory motion. A fast low-angle shot (FLASH) (TR = 1 cardiac cycle [95 - $135 \mathrm{~ms}$ ], TE $=3.2 \mathrm{~ms}$, flip angle $[\mathrm{FA}]=40^{\circ}$, and number of averages $[\mathrm{NA}]=3$ ) image was acquired to serve as anatomical reference.

The $T_{1 \rho}$ sequence consisted of $a B_{1}$ and $B_{0}$ field inhomogeneity-compensating spin-lock preparation (26), followed by a fast imaging with steady state precession (FISP) readout. The imaging acquisition window of approximately $11 \mathrm{~ms}$, preceded by the global spin-lock preparation, was kept at a constant position in the cardiac cycle to prevent motion artifacts and guarantee accurate registration between images recorded with different spinlock times. The FISP readout was performed in 22 segments of five echoes. Other sequence parameters were: TE $=1.33 \mathrm{~ms}, \mathrm{TR}=2.67 \mathrm{~ms}$, segment $\mathrm{TR}=2000 \mathrm{~ms}, \mathrm{NA}=3$, acquisition matrix $=110 \times 110$, reconstruction matrix $=$ $220 \times 220, \mathrm{FA}=30^{\circ}$. The acquisitions were performed for five different spin-lock amplitudes $\left(\gamma \mathrm{B}_{1}=100,250,500\right.$, 1000 , and $1500 \mathrm{~Hz}$ ) and with five different spin-lock times ([TSL] $=3,6,12,24$, and $48 \mathrm{~ms}$ ). Additionally, an experiment with a spin-lock amplitude of $0 \mathrm{~Hz}$ was performed to serve as a $\mathrm{T}_{2}$ measurement because under this condition it turns into a spin-echo preparation, which is $\mathrm{T}_{2}$-weighted.

After acquisition of the last preinjection image, the contrast agent bolus was injected manually. Subsequently, a second FLASH image was acquired to confirm successful intravenous injection, after which the mouse was removed from the scanner and allowed to recover. Twenty-four hours later, the mouse was anesthetized again and postinjection images were acquired. While still under anesthesia, the mice were euthanized directly after the last MRI scan.

\section{MRI Data Analysis}

Image analysis was performed in Mathematica 9.0 (Wolfram Research, Champaign, IL). Pixel-wise mono-exponential fitting of the signal intensities at different TSL was performed for each spin-lock amplitude to generate $T_{1 p}$ and $\mathrm{T}_{2}$-maps. Regions of interest (ROIs) were drawn manually around the liver and muscles on the FLASH anatomical reference images and copied to the $\mathrm{T}_{1 \mathrm{\rho}}$ and $\mathrm{T}_{2}$ maps to determine the mean liver and muscle $\mathrm{T}_{1 \rho}$ and $\mathrm{T}_{2}$, respectively. The main blood vessels of the liver and also the gall bladder, when visible in the slice, were excluded from the liver ROI. Pixels with an $\mathrm{R}^{2}$ of fit of $<0.7$ were excluded from further analysis. Histograms of $\mathrm{T}_{1 \mathrm{p}}$ and $\mathrm{T}_{2}$ in the liver were generated for each mouse with the following settings: 20 bins, bin width $5 \mathrm{~ms}$, and a range of 0 to $100 \mathrm{~ms}$. A spline function was fitted through the averaged histograms to visualize the changes in $T_{1 \rho}$ and $T_{2}$ distributions that were induced by Sinerem injection.

\section{Statistics}

Statistical analysis was performed in SPSS Statistics 22 (IBM, Armonk, NY). In vivo data on pre- and 

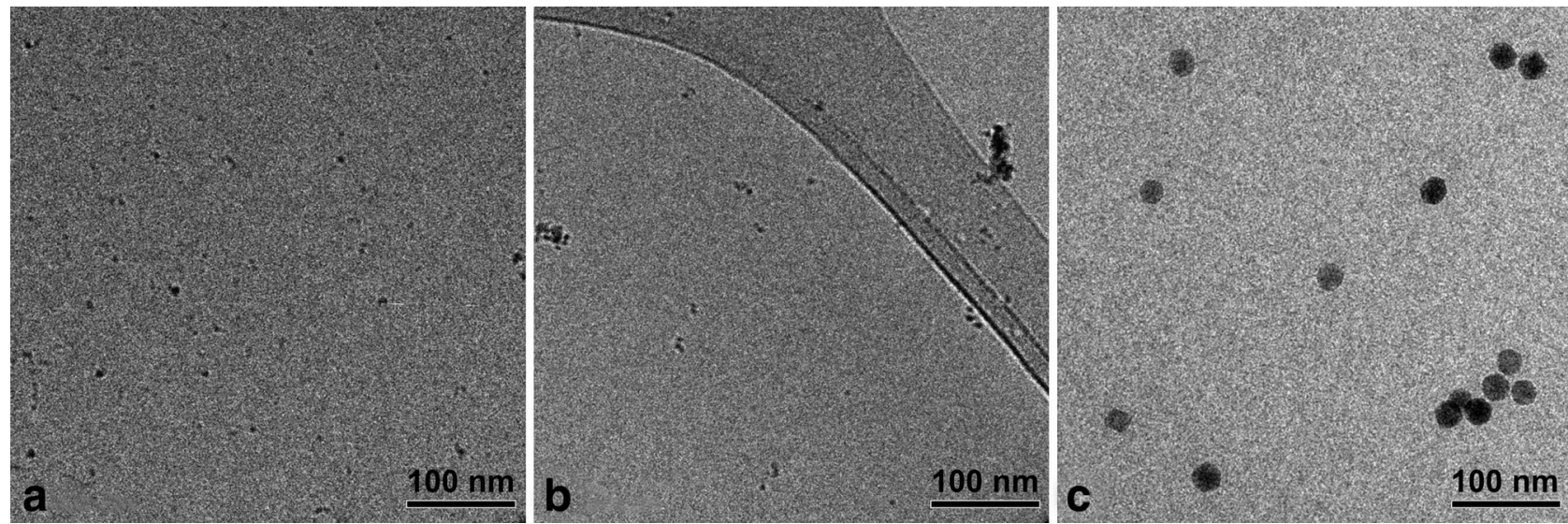

FIG. 1. Cryo- TEM images show the spatial dispersion and aggregation of the iron oxide cores of the three nanoparticles: (A) Sinerem, (B) Resovist, and (C) ION-Micelles. The particle coatings could not be observed with cryo-TEM. The background structure in (B) is part of the carbon film onto which the sample was mounted.

postinjection $T_{1 \rho}$ and $T_{2}$ values were compared with a two-sided $t$ test for each spin-lock amplitude. The effect of spin-lock amplitude on both the pre- and postinjection $\mathrm{T}_{1 \rho}$ datasets was tested for significance using an analysis of variance (ANOVA) for repeated measures with the spin-lock amplitude as the within-subject factor. In case a significant effect of spin-lock amplitude was found with ANOVA, the individual $\mathrm{T}_{1 \rho}$ levels were compared with a one-sided paired $t$ test. The level of significance was set to $\alpha=0.05$ for all tests.

\section{RESULTS}

Figure 1 shows the cryo-TEM images of the three types of nanoparticle. The iron oxide cores (black dots and discs), but not the coatings, are visible in the images. Sinerem seemed evenly dispersed with little or no aggregation of cores (Fig. 1A), which indicates that most particles contain a single core. For Resovist, the dispersion is also relatively uniform (Fig. 1B). However, alongside single cores, aggregates of two to three cores-and occasionally even larger aggregates-were observed for Resovist. This suggests that a subset of Resovist particles contain more than one iron oxide core. ION-Micelles also displayed a few small aggregates (Fig. 1C). However, the limited spacing between clustered cores indicated an aggregation of coated particles rather than single particles containing multiple cores. Higher magnification TEM images in Figure 2 show the core-shell structure of Sinerem and MPIO. Sinerem consists of spherical particles with a single core of iron oxide (Fig. 2A). MPIO are polymer beads with a broad size distribution that contain multiple iron oxide cores per particle, which can be well distinguished in the inset of Figure 2B. The saturation magnetization values for each of the contrast agents can be found in Table 1.

In Figure $3, \mathrm{~T}_{1 \rho}$ dispersion curves are shown for the in vitro experiments with different types and concentrations of iron oxides. Agar without contrast agent displayed the strongest dispersion. Over the full range of spin-lock amplitude $\gamma \mathrm{B}_{1}, \mathrm{~T}_{1 \mathrm{\rho}}$ increased from $91.7 \mathrm{~ms}$ (at $6.5 \mathrm{kHz}$ ) to $479.4 \mathrm{~ms}$ (at $91 \mathrm{kHz}$ ). The data was fitted to the exchange model for dispersion in agar gels described by Equation (2). The average exchange rate $1 / \tau_{\text {ex }}$ observed in plain agar was $143 \pm 1 \mathrm{kHz}$, which is in the range of values reported for the dominant exchanging water class in agar gels (27).

For all contrast agents, $\mathrm{T}_{1 p}$ decreased with increasing iron concentration. $\mathrm{T}_{1 \mathrm{\rho}}$ at the highest iron concentration $(500 \mu \mathrm{M})$ and maximum spin-lock amplitude $(91 \mathrm{kHz})$ was $24.2 \mathrm{~ms}$ for Sinerem (Fig. 3A), $15.6 \mathrm{~ms}$ for Resovist (Fig. 3B), $4.1 \mathrm{~ms}$ for ION-Micelle (Fig. 3C), and $233.2 \mathrm{~ms}$ for MPIO (Fig. 3D). Furthermore, in the presence of Sinerem, Resovist, and ION-Micelles, the $\mathrm{T}_{1 \mathrm{\rho}}$ dispersion curves were notably flatter. Solid lines in Figure 3 are exchange model fits using Equation (2). Extrapolated values for $T_{2}=T_{1 \rho}(0 \mathrm{kHz})$ agreed with the $T_{2}$ values as measured with the CPMG sequence (Fig. 3A-C), with the exception of MPIO, for which $\mathrm{T}_{1 \rho}(0 \mathrm{kHz})$ was higher than CPMG T 2 (Fig. 3D).

The strong reduction in $\mathrm{T}_{1 \rho}$ dispersion at higher iron oxide concentrations resulted in strong $\mathrm{T}_{1 \rho}$ differences compared to $0 \mu \mathrm{M}$ iron oxide at the higher spin-lock amplitudes. In other words, a higher $\mathrm{T}_{1 p}$ contrast is generated by the iron oxides for the higher spin-lock amplitudes. To quantify this contrast, the normalized difference in the $\mathrm{T}_{1 \rho}$ relaxation rate $\left(\mathrm{N} \Delta \mathrm{R}_{1 \rho}\right.$, Eq. [3]) as function of iron concentration for the different iron oxides and varying spin-lock amplitude is shown in Figure 4. $N \Delta R_{1 \rho}$ was highly linear with iron concentration $\left(\mathrm{R}^{2}>0.96\right)$. With the exception of MPIO, all contrast agents yielded enhanced $N \Delta R_{1 \rho}$ for increased spin-lock amplitude. $\mathrm{N} \Delta \mathrm{R}_{1 \rho}$ was enhanced up to 5.1-fold (Sinerem), 5.9-fold (Resovist), and 4.7-fold (ION-Micelle) compared to $\mathrm{N} \Delta \mathrm{R}_{2}$. Notably, for the three nanoparticles, $N \Delta R_{1 \rho}$ was larger than $N \Delta R_{2}$ - even for the smallest spinlock amplitude. For MPIO, the absolute $\mathrm{T}_{1 \rho}$ decrease was small (Fig. 3D), and a significant decrease in dispersion was not observed. This was also reflected in the very low $N \Delta R_{1 \rho}$ and $N \Delta R_{2}$ values (Fig. $4 D$ ), indicating that only minor changes in $T_{1 \rho}$ and $T_{2}$ contrast were achieved by the addition of microparticles of iron oxide.

Figures 5A,C show cross-sectional in vivo liver $\mathrm{T}_{1 \mathrm{p}^{-}}$ weighted images of mice pre- and $24 \mathrm{~h}$ postinjection of 

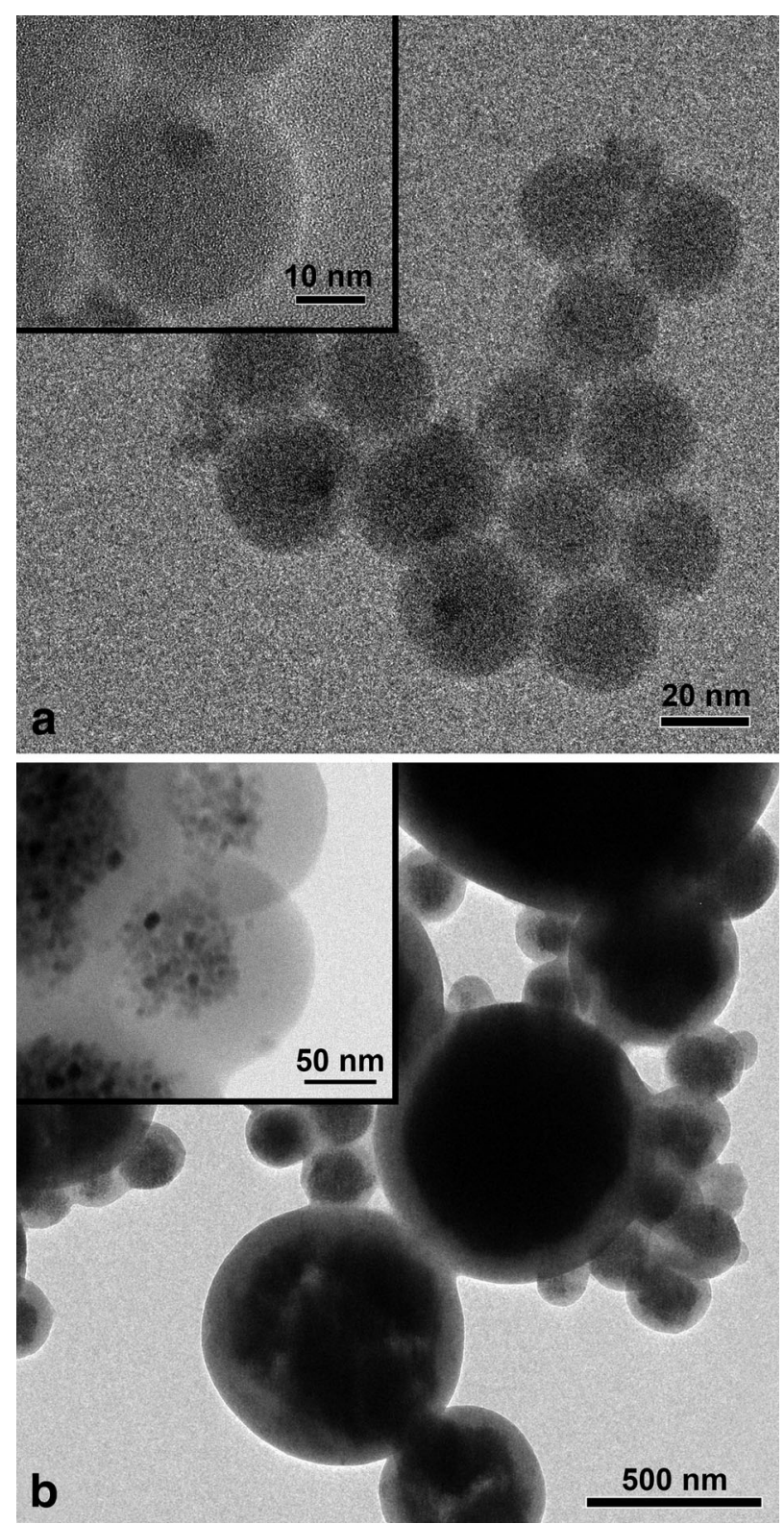

FIG. 2. TEM images of (A) Sinerem and (B) MPIO display the core shell structure of the particles. The insets show details at a higher magnification.

Sinerem. In Figures 5B,D, corresponding $\mathrm{T}_{1 \rho}$ maps for a spin-lock amplitude of $500 \mathrm{~Hz}$ are presented. Twentyfour hours after contrast agent injection, $\mathrm{T}_{1 \mathrm{p}}$-weighted signal intensity in the liver had markedly dropped, and quantitative $\mathrm{T}_{1 \rho}$ values were substantially lower. Quantitative $\mathrm{T}_{1 \rho}$ values from ROIs in the liver and muscle as a function of spin-lock amplitude are displayed in Figures $6 \mathrm{~A}, \mathrm{~B}$, respectively. Both pre- and postinjection mean liver $\mathrm{T}_{1 \rho}$ displayed a dispersion, with increasing $\mathrm{T}_{1 \rho}$ $(P<0.001)$ toward higher spin lock. Differences between pre- and post-injection $\mathrm{T}_{1 \rho}$ values were significant $(P<0.05)$ for all spin-lock amplitudes except for $100 \mathrm{~Hz}$ $(P=0.194)$ and $1500 \mathrm{~Hz}(P=0.059)$. Mean muscle $\mathrm{T}_{1 \rho}$ showed no significant dispersion with spin-lock amplitude $(P=0.357)$, and no significant differences were found between pre- and postinjection time points. All $\mathrm{T}_{2}$ values were significantly lower than the $\mathrm{T}_{1 \rho}$ values; and pre- and post- $\mathrm{T}_{2}$ values were significantly different in the liver. Figures 6C,D show typical $\mathrm{T}_{1 \rho}$ and $\mathrm{T}_{2}$ signal decay curves in liver and muscle, respectively. Apparently independent from $\mathrm{T}_{1 \mathrm{p}}$ and $\mathrm{T}_{2}$ decay, large differences in pre- and postinjection signal intensities were observed for the liver (Fig. 6C). In muscle $\mathrm{T}_{1 \rho}$ and $\mathrm{T}_{2}$ values were not influenced by injection of Sinerem. In Figure 7 , average $T_{1 \rho}$ and $T_{2}$ distributions in the liver are shown. At 24 hours after injection, a small but significant shift in the histograms toward lower relaxation values was observed for $T_{1 \rho}$ at all spin-lock amplitudes and $T_{2}$.

\section{DISCUSSION}

\section{In Vitro Experiments}

In this study, we investigated the effect of four iron oxide contrast agents on $\mathrm{T}_{1 \rho}$. In vitro experiments in agar phantoms demonstrated both a decrease in $\mathrm{T}_{1 \rho}$ values and a loss of $\mathrm{T}_{1 \rho}$ dispersion in the presence of iron oxide nanoparticles. For MPIO, no significant changes in $\mathrm{T}_{1 \rho}$ dispersion were observed. Due to the loss of dispersion, a higher contrast was generated at high spin-lock amplitudes. The normalized contrast $\left(\mathrm{N} \Delta \mathrm{R}_{1 \rho}\right)$ proved linear with iron concentration. For all three nanoparticle contrast agents, $N \Delta R_{1 \rho}$ was higher than $N \Delta R_{2}$ and was found to increase with spin-lock amplitude. The fact that the extrapolated values for $\mathrm{T}_{2}=\mathrm{T}_{1 \rho}(0 \mathrm{~Hz})$ matched the $\mathrm{T}_{2}$ values measured by CPMG is in accordance with $\mathrm{T}_{1 \rho}$ relaxation theory (15).

\section{Iron Oxide $\mathrm{T}_{1 \rho}$ Contrast Mechanism}

We believe that the mechanism underlying enhanced iron oxide $\mathrm{T}_{1 \rho}$ contrast is loss of spin lock, leading to the reduction of native tissue $\mathrm{T}_{1 \rho}$ dispersion. The effect of iron oxides on $\mathrm{T}_{2}\left({ }^{*}\right)$ relaxation has been extensively studied, and the effects of particle size and coating composition are well understood (21-24). Important factors for the $R_{2}{ }^{*}$ and $R_{2}$ relaxation rates are the strength and spatial extent of local field gradients, proton diffusion during the time of the NMR experiment, and the relative proportion of these factors. Several relaxation regimes can be distinguished, but the discussion here will focus on those regimes relevant for the present experiments. For a full discussion of all $\mathrm{R}_{2}{ }^{*}$ ) regimes, we refer to refs. [22) and (23).

The first regime that is of importance for the iron oxide $\mathrm{T}_{1 \rho}$ contrast mechanism is the static dephasing regime (SDR). In this regime, which is generally applicable for large particles, proton displacement by diffusion is small relative to the spatial scale of field gradients. Hence, protons near the particle essentially experience a static field offset. For $\mathrm{R}_{2}$ * relaxation, this means rapid dephasing and $R_{2}{ }^{*}$ approaches a maximum. $R_{2}$ is low because the static field offset can be effectively compensated through a refocusing pulse. In the case of $R_{1 \rho}$ during spin-locking, a static field offset will cause offresonance spin lock along an effective magnetic field

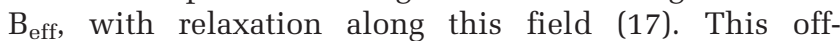
resonance relaxation rate $R_{1 \rho}$ off becomes a combination of $R_{1}$ and $R_{1 \rho}$ in a ratio dependent on the ratio of $B_{1}$ and 

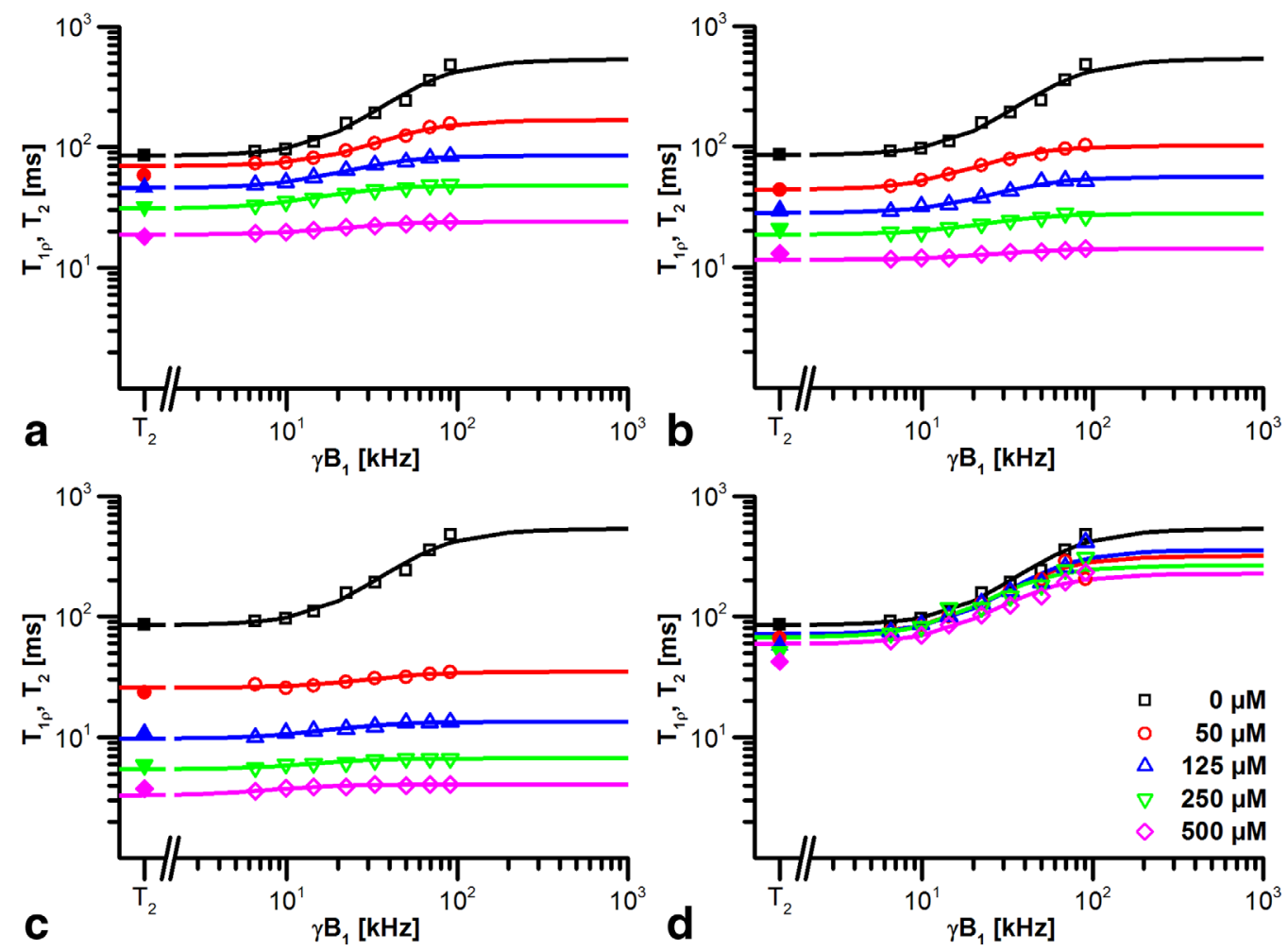

FIG. 3. In vitro $T_{1 \rho}$ dispersion curves. $T_{1 \rho}$ as function of $\gamma B_{1}$ for different concentrations of (A) Sinerem, (B) Resovist, (C) ION-Micelle, and (D) MPIO in $2 \%$ agar gel. Solid lines are exchange model fits using Eq. [2], which represents the native $T_{1 \rho}$ dispersion of agar. Both $T_{1 \rho}$ values and $T_{1 \rho}$ dispersion decreased with increasing iron concentration. The data points located to the left of the horizontal axis break (solid symbols) are $T_{2}$ values measured by CPMG.
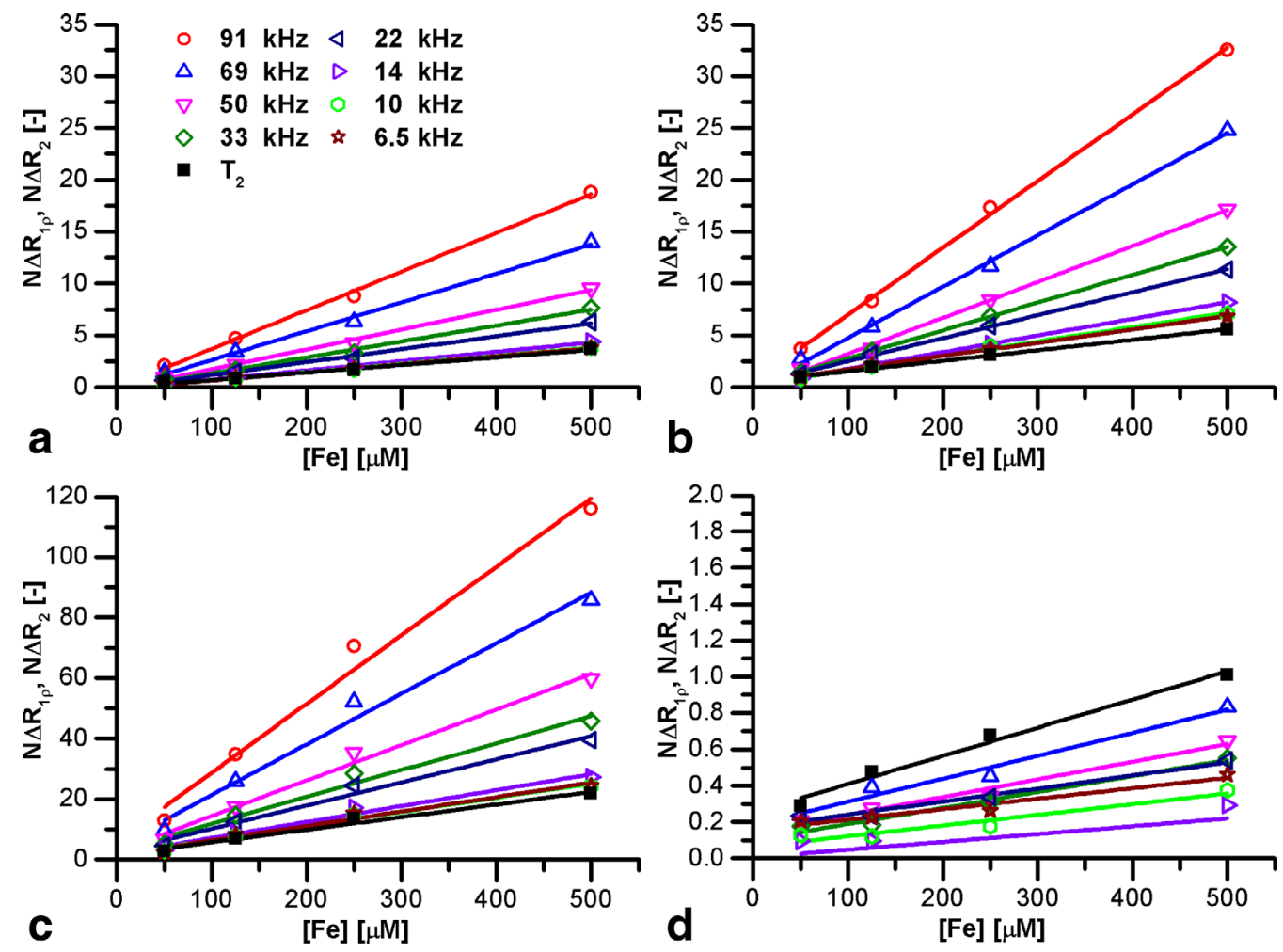

FIG. 4. $T_{1 \rho}$ contrast. $N \Delta R_{1 \rho}$ as a function of iron concentration [Fe] for varying spin-lock amplitudes for (A) Sinerem, (B) Resovist, (C) ION-Micelle, and (D) MPIO. The solid symbols are $N \Delta R_{2}$. Solid lines are linear fits of the data $\left(R^{2}>0.96\right)$. For all iron oxides, $N \Delta R_{1 \rho}$ increased linearly with iron concentration. $N \Delta R_{1 \rho}$ was higher than $N \Delta R_{2}$ for all preparations, with the exception of MPIO. Note that the vertical axes have different scaling. 


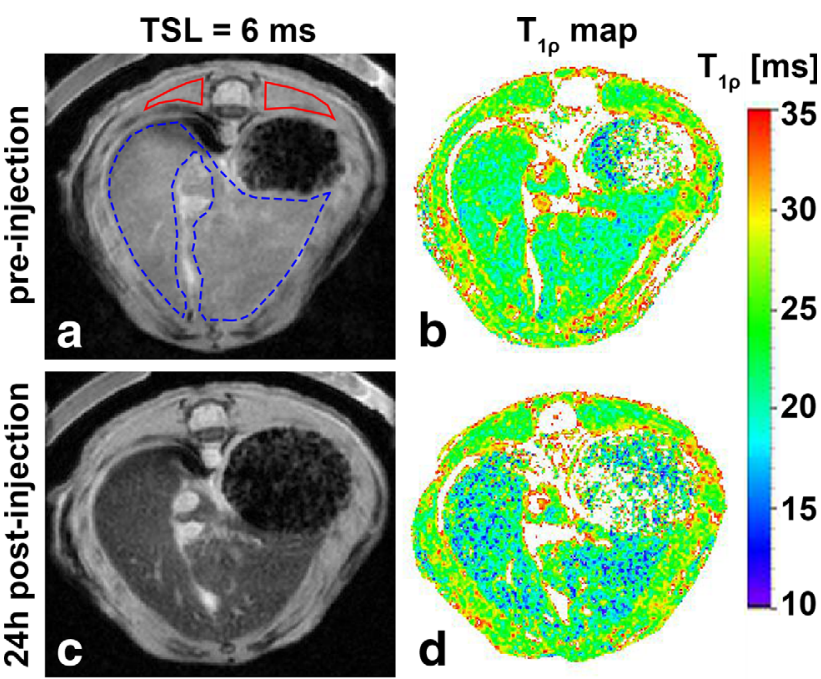

FIG. 5. In vivo $T_{1 \rho}$ imaging of mouse liver pre- and post-Sinerem injection. Examples from a single mouse of: (A) pre- and (C) postinjection $\mathrm{T}_{1 \mathrm{p}}$-weighted images acquired with spin-lock amplitude $500 \mathrm{~Hz}$ and TSL $=6 \mathrm{~ms}$, and (B) pre- and (D) postinjection $T_{1 \mathrm{p}}$ maps acquired by pixel-wise fitting of the signal intensities of all TSL images with spin-lock amplitude $500 \mathrm{~Hz}$. In (A), solid red lines are ROls in muscle; the dashed blue line is the ROI in the liver.

$\Delta \omega$ (20). Off-resonance spin lock is often applied to decrease specific absorption rate (SAR) because a similar $B_{\text {eff }}$ can be reached with a lower $B_{1}$ (19). In this case, however, the source of off-resonance is the contrast agent and not the RF pulse. Because $B_{1}$ remains unchanged, a higher $B_{\text {eff }}$ is achieved in the vicinity of the particle. The field offset is static; therefore, no loss of spin lock and thus no effect on the native $\mathrm{T}_{1 \rho}$ dispersion of a tissue are to be expected in the SDR.

The other regime that is considered important for the experiments in this study is the so-called visit-limited regime (VLR); it marks the transition between the SDR and the motional averaging regime, which will not be discussed here (22). Iron oxide nanoparticles in the VLR are surrounded by a full dephasing zone in which the proton spins are fully dephased upon entry. According to our hypothesis, the gradients and diffusional motion will lead to a loss of spin lock in the full dephasing zone. Experimental observations (Figs. 3 and 4) are in agreement with this hypothesis. First, strong dephasing in the full dephasing zone in the presence of the iron oxide nanoparticles leads to a rapid decrease in signal intensity as a function of TSL, resulting in a lower measured $\mathrm{T}_{1 \rho}$ value. Furthermore, because of the loss of spin lock during TSL, signal decay and thus $\mathrm{T}_{1 \rho}$ become essentially independent from the spin-lock amplitude, resulting in a loss of the exchange-mediated dispersion with $\gamma B_{1}$ of native agar. Moreover, $N \Delta R_{1 \rho}$ was linear with concentration, which can be explained by a linear increase of the total volume occupied by the full dephasing zones surrounding the particles with increasing concentration.
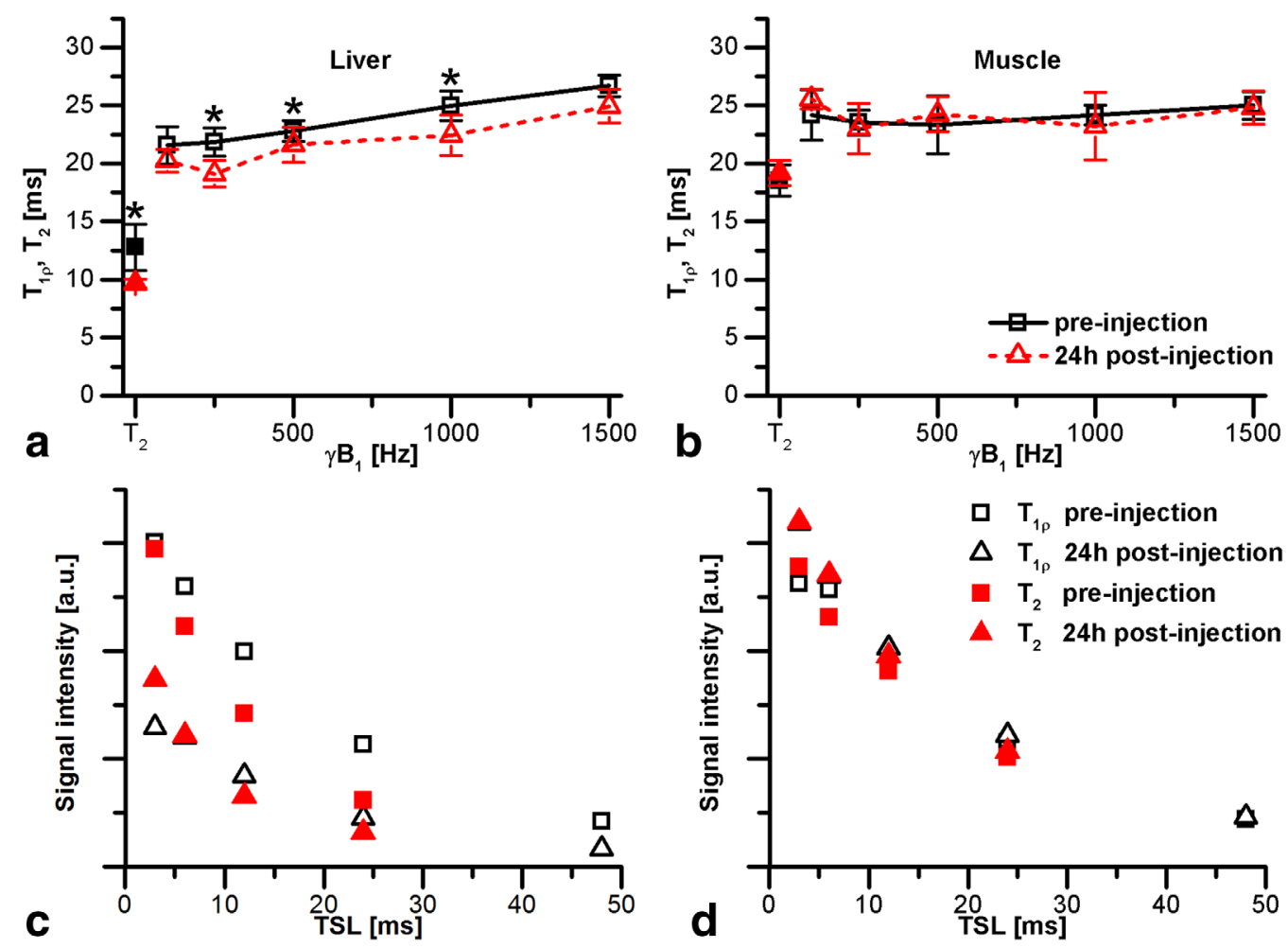

FIG. 6. In vivo $T_{1 \rho}$ contrast. Top row: mean quantitative $T_{1 \rho}$ and $T_{2}$ of $(A)$ mouse liver and (B) muscle pre- and postinjection of Sinerem. Symbols indicate pre- and postinjection $T_{1 \rho}$ (open symbols) and $T_{2}$ (solid symbols) values versus spin-lock amplitude averaged over all mice $(\mathrm{n}=7)$. Error bars are standard deviations, ${ }^{*}$ indicates significant differences between pre- and postinjection $(P<0.05)$. Bottom row: typical pre- and postinjection signal decay curves of (C) liver and (D) muscle of one mouse. $\mathrm{T}_{1 \mathrm{p}}$-weighted signal intensity at $500 \mathrm{~Hz}$ (open symbols) and $\mathrm{T}_{2}$-weighted signal intensity (solid symbols) are plotted as a function of TSL. 

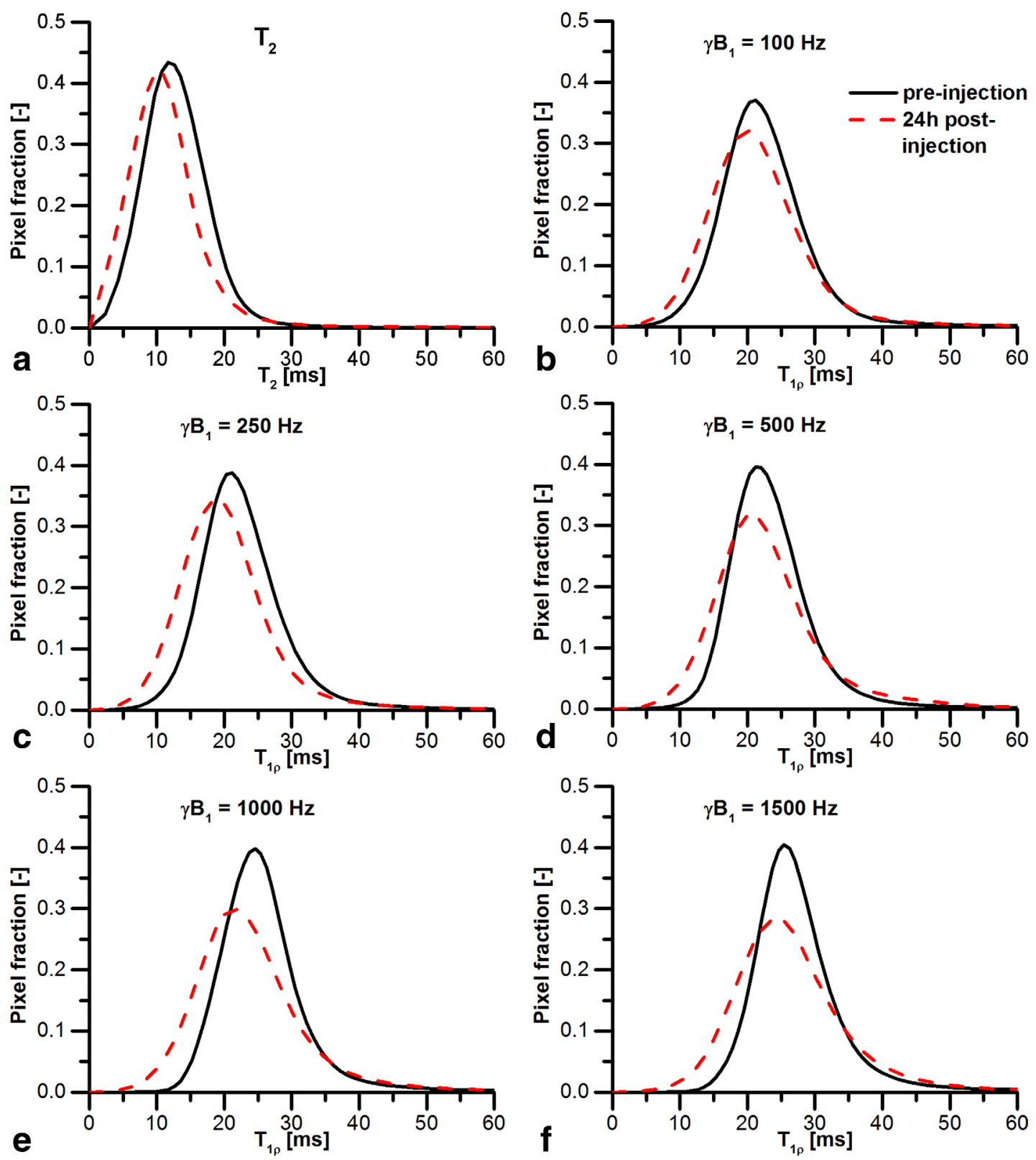

FIG. 7. Average $T_{2}$ and $T_{1 \rho}$ distributions in the mouse liver before and $24 \mathrm{~h}$ after Sinerem injection. The average pre- and postinjection (A) $T_{2}$ and (B-F) $T_{1 \rho}$ distributions at all spin-lock amplitudes were constructed by spline fitting of averaged histograms.

The VLR is applicable to particles at the transition between the motional averaging regime and the SDR. This transition is defined by

$$
\frac{\mathrm{r}_{\mathrm{p}}^{2}}{\mathrm{D}} \approx \frac{\pi \sqrt{15}}{4 \Delta \omega_{\mathrm{r}}}
$$

with $r_{p}$ the radius of a nanoparticle in the VLR, D the diffusion coefficient, and $\Delta \omega_{\mathrm{r}}$ the root-mean-squared frequency shift at the particle surface (28).

The diffusion coefficient in a $2 \%$ agar gel is of the same order of magnitude as that of free water $(\sim 2.5$. $10^{-9} \mathrm{~m}^{2} / \mathrm{s}$ ), and the frequency shift at the particle surface caused by its magnetization is on the order of that of magnetite $\left(3.0 \cdot 10^{7} \mathrm{rad} / \mathrm{s}\right)$. This means that relaxation of water in the proximity of particles with a diameter of around $30 \mathrm{~nm}$ occurs in the visited limiting regime $(22,28)$. With its much larger diameter of $860 \mathrm{~nm}$, MPIO is therefore in the SDR. With hydrodynamic diameters between approximately 30 and $60 \mathrm{~nm}$ (Table 1), the VLR applies to Sinerem, Resovist, and ION-Micelles (23).

\section{Comparison of the Four Contrast Agents}

Comparing different nanoparticles, $N \Delta \mathrm{R}_{1 \mathrm{\rho}}$ was higher for Resovist than for Sinerem (Fig. 4). Differences in the observed $\mathrm{T}_{1 \rho}$ contrast between the different iron oxide formulations can be related to their different physicochemical properties (Table 1). Sinerem and Resovist have comparable iron oxide core sizes, and both have similar dextran-based coatings. However, the coating of Resovist is twice as thick as the one of Sinerem. 
Therefore, the full dephasing zone of Resovist may occupy a larger volume, leading to a stronger $T_{1 \rho}$ decrease and more pronounced loss of dispersion as compared to Sinerem (23). Also, the saturation magnetization for Resovist is higher (89 vs. $71 \mathrm{Am}^{2} \mathrm{~kg}^{-1} \mathrm{Fe}$ ). Additionally, Resovist has a broader size distribution than Sinerem and contains a small fraction ( $~ 3 \%)$ of particles with a larger iron core up to $30 \mathrm{~nm}(29,30)$. Also, multiple cores per particle (Fig. 1B) are found, leading to an effectively higher magnetic moment per particle, and therefore stronger $\mathrm{T}_{1 \rho}$ decrease with concentration.

ION-Micelles displayed the highest $N \Delta R_{1 \rho}$ of the four iron oxide formulations. The main reason can be found in the larger size of the particle's core and the higher magnetic moment per particle. The monocrystalline core of ION-Micelles is approximately five times larger in diameter $(25 \mathrm{~nm})$ than those of Sinerem and Resovist (both 4-6 nm). A larger core combined with a higher saturation magnetization (93 $\mathrm{Am}^{2} \mathrm{~kg}^{-1} \mathrm{Fe}$ ) results in a much larger magnetic moment per particle, with larger local field gradients and a larger affected volume for each individual particle. The amphiphilic phospholipid coating with hydrophilic polyethylene-glycol (PEG) tails constitute a region of restricted diffusion similar to the dextran for Sinerem and Resovist (31). However, this coating is thinner than the dextran coatings; therefore, the main reason for the larger $N \Delta R_{1 \rho}$ is probably the much larger magnetic moment of ION-Micelles.

For MPIO, a minor $\mathrm{N} \Delta \mathrm{R}_{1 \rho}$ and no significant change in $\mathrm{T}_{1 \rho}$ dispersion were observed. MPIO consists of multiple small iron oxide cores incorporated in a polymer mesh with an outer layer of pure polymer (Fig. 2B). The particles can be regarded as having one very large $(860 \mu \mathrm{m})$ superparamagnetic core (28), producing an equally large magnetic moment. The polymer mesh is not water permeable; thus, water protons are restricted to the outer regions of the gradient field where the magnetic moment is lower and there is less spatial fluctuation than close to the center. These particles are in the static-dephasing regime; rather than inducing a loss of spin lock, they will preserve off-resonance spin lock and $\mathrm{T}_{1 \rho}$ dispersion. Also, because of their lower surface-area-to-volume ratio (circa 0.007 vs. 0.1-0.2 for the nanoparticles), a much smaller effective volume, and thus a smaller portion of protons in the sample, are affected. Additionally, because the iron concentration was kept constant for different contrast agents and the MPIOs have a larger iron load, the particle concentration was much lower, which further adds to a smaller $\mathrm{T}_{1 \rho}$ change.

\section{In Vivo Iron Oxide $T_{1 \rho}$ Contrast}

The in vivo experiments revealed minor $\mathrm{T}_{1 \rho}$ dispersion in the mouse liver both before and 24 hours after intravenous injection of a bolus of Sinerem (Fig. 6A). The $T_{1 \rho}$ distribution in the liver shifted toward lower values after injection for all spin-lock amplitudes (Fig. 7), yielding a significantly decreased mean liver $\mathrm{T}_{1 \rho}$ at spin-lock amplitudes of 250, 500, and $1000 \mathrm{~Hz}$. These changes of in vivo $T_{1 \rho}$ values were only small and were not significantly different from the reduction of in vivo $\mathrm{T}_{2}$ values.
The in vitro advantage of $\mathrm{T}_{1 \rho}$ over $\mathrm{T}_{2}$ was thus not observed in vivo for Sinerem accumulation in the liver.

In vivo $\mathrm{T}_{1 \rho}$ dispersion in the liver did not appear to be influenced by the presence of Sinerem in a similar fashion as for the agar experiments. One explanation for this may be found in the in vivo fate of the particles. Sinerem is mainly taken up by Kupffer cells in the liver, resulting in a clustered distribution rather than the even dispersion in agar gels in vitro (32). Similar to the clustering of iron oxide cores inside the MPIO particles, these clusters might behave as larger particles with a SDR relaxation mechanism (28). Indeed, it is known that such in vivo clustering results in reduced changes in $R_{1}$ and $R_{2}$ values $(32,33)$. Another cause may be found in the low precontrast dispersion, which makes it difficult to detect significant dispersion changes. Additionally, due to hardware restrictions, only a low spin-lock amplitude (1500 Hz) could be applied in vivo, limiting the range over which the dispersion could be studied.

The liver contrast between pre- and postinjection in the $\mathrm{T}_{1 \mathrm{p}}$-weighted images appeared larger than what could be expected on the basis of quantitative $\mathrm{T}_{1 \rho}$ differences (Fig. 5). The reason is a $\mathrm{T}_{2}{ }^{*}$ effect in the FISP imaging readout, which leads to a shift of the whole $\mathrm{T}_{1 \rho}$ and $\mathrm{T}_{2}$ relaxation curves toward lower signal intensity values (Fig. 6C), independent of changes in the quantitative $T_{1 \rho}$ and $\mathrm{T}_{2}$ values.

The fact that no loss of $\mathrm{T}_{1 \rho}$ dispersion was observed in the liver does not preclude in vivo application of $T_{1 \rho}$ imaging of iron oxide contrast agents in other tissues such as articular cartilage $(34,35)$, myocardial tissue (36), breast tissue (37), and certain tumors (38) that display larger endogenous $\mathrm{T}_{1 \rho}$ dispersions. Furthermore, techniques such as adiabatic $\mathrm{T}_{1 \rho}$ and relaxation along a fictitious field $(35,39-41)$ may enable higher effective spinlock amplitudes to allow probing of higher frequency dispersion regimes without violating hardware restrictions. These techniques also have the advantage that they lower the SAR, which hampers clinical application of conventional $\mathrm{T}_{1 \rho}$ imaging. However, the effects of iron oxides on $\mathrm{T}_{1 \rho}$ behavior using these sequences remain to be investigated.

\section{CONCLUSION}

In the in vitro experiments, it was proven that iron oxide nanoparticles cause loss of spin lock, resulting in the suppression of $\mathrm{T}_{1 \rho}$ dispersion. Thereby, $\mathrm{T}_{1 \rho}$ contrast is enhanced compared to $\mathrm{T}_{2}$. Spin-lock MR is therefore a promising technique for the sensitive detection of iron oxide contrast agents. However, evaluation in the mouse liver did not reveal an improvement in $\mathrm{T}_{1 \rho}$ sensitivity above $\mathrm{T}_{2}$ for iron oxide in vivo, which was probably due to a lack of initial $\mathrm{T}_{1 \rho}$ dispersion in the liver and limitations in available spin-lock power.

\section{ACKNOWLEDGMENTS}

The authors thank Holger Grüll, Monja Kaiser, and Marcel Verheijen for (cryo-)TEM measurements; Erica Aussems-Custers for support with the ION-Micelle synthesis; Reinoud Lavrijsen for vibrating sample 
magnetometer measurements; and Jeanette Smulders for ICP-AES measurements.

\section{REFERENCES}

1. McAteer MA, Akhtar AM, von Zur Muhlen C, Choudhury RP. An approach to molecular imaging of atherosclerosis, thrombosis, and vascular inflammation using microparticles of iron oxide. Atherosclerosis 2010;209:18-27.

2. Sosnovik D, Nahrendorf M, Weissleder R. Magnetic nanoparticles for MR imaging: agents, techniques and cardiovascular applications. Basic Res Cardiol 2008;103:122-130.

3. Laurent S, Boutry S, Mahieu I, Vander Elst L, Muller RN. Iron oxide based MR contrast agents: from chemistry to cell labeling. Curr Med Chem 2009;16:4712-4727.

4. Laurent S, Forge D, Port M, Roch A, Robic C, Vander Elst L, Muller RN. Magnetic iron oxide nanoparticles: synthesis, stabilization, vectorization, physicochemical characterizations, and biological applications. Chem Rev 2008;108:2064-2110.

5. Moonen RPM, Nicolay K, Strijkers GJ. Quantification of USPIO uptake in mouse atherosclerotic plaque by T2 mapping MRI. Magn Reson Mater Physics Biol Med ESMRMB 2012, 29th Annual Sci Meeting, Lisbon, Portugal. 4-6 October. Abstract. 2012;25: Suppl 1; 73 p55-56.

6. Coolen BF, Simonis FFJ, Geelen T, Moonen RPM, Arslan F, Paulis LEM, Nicolay K, Strijkers GJ. Quantitative T2 mapping of the mouse heart by segmented MLEV phase-cycled T2 preparation. Magn Reson Med 2014;72:409-417.

7. Sadat U, Howarth SPS, Usman A, Tang TY, Graves MJ, Gillard JH. Sequential imaging of asymptomatic carotid atheroma using ultrasmall superparamagnetic iron oxide-enhanced magnetic resonance imaging: a feasibility study. J Stroke Cerebrovasc Dis 2013;22:e271-e276.

8. Degnan AJ, Patterson AJ, Tang TY, Howarth SPS, Gillard JH. Evaluation of ultrasmall superparamagnetic iron oxide-enhanced MRI of carotid atherosclerosis to assess risk of cerebrovascular and cardiovascular events: follow-up of the ATHEROMA trial. Cerebrovasc Dis 2012;34:169-173.

9. Patterson AJ, Tang TY, Graves MJ, Müller KH, Gillard JH. In vivo carotid plaque MRI using quantitative T2* measurements with ultrasmall superparamagnetic iron oxide particles: a dose-response study to statin therapy. NMR Biomed 2011;24:89-95.

10. Kuhlpeter R, Dahnke H, Matuszewski L, Persigehl T, von Wallbrun A, Allkemper T, Heindel WL, Schaeffter T, Bremer C. R2 and R2* Mapping for sensing cell-bound superparamagnetic nanoparticles: in vitro and murine in vivo testing. Radiology 2007;245:449-457.

11. Cobb JG, Xie J, Gore JC. Contributions of chemical exchange to T1 $\rho$ dispersion in a tissue model. Magn Reson Med 2011;66:1563-1571.

12. Cobb JG, Xie J, Gore JC. Contributions of chemical and diffusive exchange to T1 $\rho$ dispersion. Magn Reson Med 2013;69:1357-1366.

13. Borthakur A, Wheaton AJ, Gougoutas AJ, Akella SVS, Regatte RR, Charagundla SR, Reddy R. In vivo measurement of T1rho dispersion in the human brain at 1.5 tesla. J Magn Reson Imaging 2004;19:403-409.

14. Engelhardt RT, Johnson GA. T1 $\rho$ relaxation and its application to MR histology. Magn Reson Med 1996;35:781-786.

15. Santyr GE, Henkelman RM, Bronsiull MJ. Variation in measured transverse relaxation in tissue resulting from spin locking with the CPMG sequence. J Magn Reson 1988;79:28-44.

16. Mäkelä HI, Gröhn OH, Kettunen MI, Kauppinen RA. Proton exchange as a relaxation mechanism for $\mathrm{T} 1$ in the rotating frame in native and immobilized protein solutions. Biochem Biophys Res Commun 2001; 289:813-818.

17. Santyr GE, Fairbanks EJ, Kelcz F, Sorenson JA. Off-resonance spin locking for MR imaging. Magn Reson Med 1994;32:43-51.

18. Martirosian P, Rommel E, Schick F, Deimling M. Control of susceptibility-related image contrast by spin-lock techniques. Magn Reson Imaging 2008;26:1381-1387.

19. Witschey WRT, Borthakur A, Elliott MA, Mellon E, Niyogi S, Wang C, Reddy R. Compensation for spin-lock artifacts using an off-resonance rotary echo in T1poff-weighted imaging. Magn Reson Med 2007;57:2-7.

20. Gröhn OHJ, Mäkelä HI, Lukkarinen JA, DelaBarre L, Lin J, Garwood M, Kauppinen RA. On- and off-resonance T(1rho) MRI in acute cerebral ischemia of the rat. Magn Reson Med 2003;49:172-176.

21. Muller RN, Gillis P, Moiny F, Roch A. Transverse relaxivity of particulate MRI contrast media: from theories to experiments. Magn Reson Med 1991;22:178-182; discussion 195-196.
22. De Haan HW. Mechanisms of proton spin dephasing in a system of magnetic particles. Magn Reson Med 2011;66:1748-1758.

23. De Haan HW, Paquet C. Enhancement and degradation of the R2* relaxation rate resulting from the encapsulation of magnetic particles with hydrophilic coatings. Magn Reson Med 2011;66:1759-1766.

24. Strijkers GJ, Nicolay K. Relaxivity of nanoparticles for magnetic resonance imaging. In: Sattler KD, ed. Handbook of Nanophysics: Nanomedicine and Nanorobotics. Boca Raton, FL: CRC Press; 2011; 1-23.

25. Starmans LWE, Burdinski D, Haex NPM, Moonen RPM, Strijkers GJ, Nicolay K, Grüll H. Iron oxide nanoparticle-micelles (ION-micelles) for sensitive (molecular) magnetic particle imaging and magnetic resonance imaging. PLoS One 2013;8:e57335.

26. Witschey WRT, Borthakur A, Elliott MA, Mellon E, Niyogi S, Wallman DJ, Wang C, Reddy R. Artifacts in T1 rho-weighted imaging: compensation for $\mathrm{B}(1)$ and $\mathrm{B}(0)$ field imperfections. J Magn Reson 2007;186:75-85.

27. Chávez FV, Halle B. Molecular basis of water proton relaxation in gels and tissue. Magn Reson Med 2006;56:73-81.

28. Matsumoto Y, Jasanoff A. T2 relaxation induced by clusters of superparamagnetic nanoparticles: Monte Carlo simulations. Magn Reson Imaging 2008;26:994-998.

29. Chen D-X, Sun N, Gu H-C. Size analysis of carboxydextran coated superparamagnetic iron oxide particles used as contrast agents of magnetic resonance imaging. J Appl Phys 2009;106:063906.

30. Gleich B, Weizenecker J. Tomographic imaging using the nonlinear response of magnetic particles. Nature 2005;435:1214-1217.

31. LaConte LEW, Nitin N, Zurkiya O, Caruntu D, O’Connor CJ, Hu X, Bao G. Coating thickness of magnetic iron oxide nanoparticles affects R2 relaxivity. J Magn Reson Imaging 2007;26:1634-1641.

32. Tanimoto A, Oshio K, Suematsu M, Pouliquen D, Stark DD. Relaxation effects of clustered particles. J Magn Reson Imaging 2001;14:72-77.

33. Lévy M, Wilhelm C, Devaud M, Levitz P, Gazeau F. How cellular processing of superparamagnetic nanoparticles affects their magnetic behavior and NMR relaxivity. Contrast Media Mol Imaging 2012;7: 373-383.

34. Regatte RR, Akella SVS, Borthakur A, Reddy R. Proton spin-lock ratio imaging for quantitation of glycosaminoglycans in articular cartilage. J Magn Reson Imaging 2003;17:114-121.

35. Ellermann J, Ling W, Nissi MJ, Arendt E, Carlson CS, Garwood M, Michaeli S, Mangia S. MRI rotating frame relaxation measurements for articular cartilage assessment. Magn Reson Imaging 2013;31:15371543.

36. Witschey WRT, Pilla JJ, Ferrari G, Koomalsingh K, Haris M, Hinmon R, Zsido G, Gorman JH, Gorman RC, Reddy R. Rotating frame spin lattice relaxation in a swine model of chronic, left ventricular myocardial infarction. Magn Reson Med 2010;64:1453-1460.

37. Santyr GE, Henkelman RM, Bronskill MJ. Spin locking for magnetic resonance imaging with application to human breast. Magn Reson Med 1989;12:25-37.

38. Hectors SJCG, Moonen RPM, Strijkers GJ, Nicolay K. T1 $\rho$ mapping for the evaluation of high intensity focused ultrasound tumor treatment. Magn Reson Med 2014;00:1-9.

39. Deschamps M, Kervern G, Massiot D, Pintacuda G, Emsley L, Grandinetti PJ. Superadiabaticity in magnetic resonance. J Chem Phys 2008;129:204110.

40. Liimatainen T, Sorce DJ, O’Connell R, Garwood M, Michaeli S. MRI contrast from relaxation along a fictitious field (RAFF). Magn Reson Med 2010;64:983-994.

41. Mangia S, Liimatainen T, Garwood M, Michaeli S. Rotating frame relaxation during adiabatic pulses vs. conventional spin lock: simulations and experimental results at 4 T. Magn Reson Imaging 2009;27: 1074-1087.

42. Laurent S, Vander Elst L, Muller RN. Superparamagnetic iron oxide nanoparticles for MRI. In: Merbach A, Helm L, Toth E, eds. The Chemistry of Contrast Agents in Medical Magnetic Resonance Imaging. 2nd ed. Hoboken, NJ: John Wiley \& Sons; 2013; 427-447. [WorldCat]

43. Cengelli F, Maysinger D, Tschudi-Monnet F, Montet X, Corot C, Petri-Fink A, Hofmann H, Juillerat-Jeanneret L. Interaction of functionalized superparamagnetic iron oxide nanoparticles with brain structures. J Pharmacol Exp Ther 2006;318:108-116.

44. Reimer P, Rummeny EJ, Daldrup HE, Balzer T, Tombach B, Berns T, Peters PE. Clinical results with Resovist: a phase 2 clinical trial. Radiology 1995;195:489-496. 